\title{
A new method for handover triggering condition estimation
}

\author{
Shafayat Abrar ${ }^{1}$, Riaz Hussain ${ }^{1}$, Raja A. Riaz ${ }^{1,2,3}$, \\ Shahzad A. Malik ${ }^{1}$, Shahid A. Khan ${ }^{1}$, Ghufran Shafiq ${ }^{1,2}$, \\ and Saeed Ahmed ${ }^{1,2 a)}$ \\ ${ }^{1}$ Department of Electrical Engineering, COMSATS Institute of Information \\ Technology, Islamabad, Pakistan \\ ${ }^{2}$ Center for Advanced Studies in Telecommunication (CAST), COMSATS Institute \\ of Information Technology, Islamabad, Pakistan \\ ${ }^{3}$ School of ECS, University of Southampton, SO17 1BJ, UK \\ a)saeed_comsats@comsats.edu.pk
}

\begin{abstract}
It is desirable to maximize the WLAN usage in integrated heterogeneous network environment due to its high speed access and low access cost. We have modeled under geometry and mobility effects to dynamically estimate the distance of a mobile terminal from the access point at which the handover must be triggered to keep probability of handover failure within desired bounds while maximizing the WLAN usage. Monte-Carlo simulations are provided and they are in good conformance with our analytical findings.
\end{abstract}

Keywords: handover failure probability, connection breakdown probability, vertical handover decision

Classification: Wireless communication hardware

\section{References}

[1] S. Mohanty, "A new architecture for 3G and WLAN integration and intersystem handover management," Wireless Networks, vol. 12, pp. 733-745, 2006.

[2] S. Mohanty and I. F. Akyildiz, "A cross-layer (layer 2+3) handover management protocol for next-generation wireless systems," IEEE Trans. Mobile Comput., vol. 5, pp. 1347-1360, 2006.

[3] M. Ruggieri, F. Graziosi, and F. Santucci, "Modeling of the handover dwell time in cellular mobile communications systems," IEEE Trans. Veh. Technol., vol. 47, no. 2, pp. 489-498, 1998.

[4] X. Yan, Optimization of Vertical handover Decision Processes for Fourth Generation Heterogeneous Wireless Networks, PhD thesis, Australia, Monash University, 2010.

[5] F. A. Haight, "Some probability distributions associated with commuter travel in a homogeneous circular city," Operations Research, vol. 12, no. 6, pp. 964-975, 1964.

[6] F. P. Fontan and P. M. Espineira, Modeling the Wireless Propagation Channel: A Simulation Approach with MATLAB, Wiley, 2002. 


\section{Introduction}

Efficient mobility management in heterogeneous network environment can enhance the freedom of the ever-demanding mobile user. Not only the coverage area can be extended, but the user can achieve the always best connectivity in terms of quality and cost. In order to get the maximum benefit from the most desired network, it is required to ensure that handover is triggered at an appropriate instance. Not only that connectivity with the most desired network is maximized, but also the handover failure is maintained at an acceptable level.

WLAN is usually the choice from the available networks due to its high speed and low cost. However, due to its small coverage area, the high speed mobile user can benefit from WLAN to a very limited extent. There is a need to optimize the handover trigger condition for an MT moving out of the WLAN coverage area. The distance, a moving out MT would cover in the coverage area, after the handover is triggered, is critical distance. If the the handover signalling is not completed with in this critical distance the transfer of the session to the target network would result in a failure. In [1], Mohanty derived the probability density function (pdf) of critical distance under the assumption that the boundary region is square in shape and the angle of departure is uniformly distributed over that square region. In another work [2], Mohanty and Akyildiz derived the pdf of critical distance under the assumption that the boundary region is triangular in shape and the angle of departure is uniformly distributed. In this work, we obtain the pdf of critical distance in a circular WLAN region without making assumption for the pdf of angle of departure. Our analysis leads to a new method for handover triggering condition estimation. This study also reveals that the choice of the pdf of departure angle, which is not known in real scenarios, has a very little impact on overall performance.

\section{Modeling Under Geometry and Mobility Effects}

The geometrical configuration of a WLAN is presented in Fig. 1 where Access Point (AP) is located at the origin $A(0,0)$. The radius $r$ represents the distance between an AP and the point where an MT, moving along a straight line with constant velocity $v$, experiences a signal power $S_{\text {ho }}$ (handover threshold) and issues a handover request. We assume that the MT may enter the boundary area at any point on the perimeter of radius $r$ with equal probability. The outer circle of radius $R$ corresponds to the lowest signal power $S_{\text {qt }}$ (quality threshold) required for the MT to maintain connectivity with the AP. The difference $\Delta=R-r$ is the depth of handover area along the radius. The region within WLAN coverage area in which the signal power is above $S_{\text {ho }}$ is referred to as efficient region and it is called critical region otherwise.

The MT enters at point $P_{i}$ and exits at point $P_{o}$ and maintains a straight line motion in the coverage area. When the MT is at the perimeter of radius $r$ at point $C$, it moves out of the coverage region through the arc EF along a 


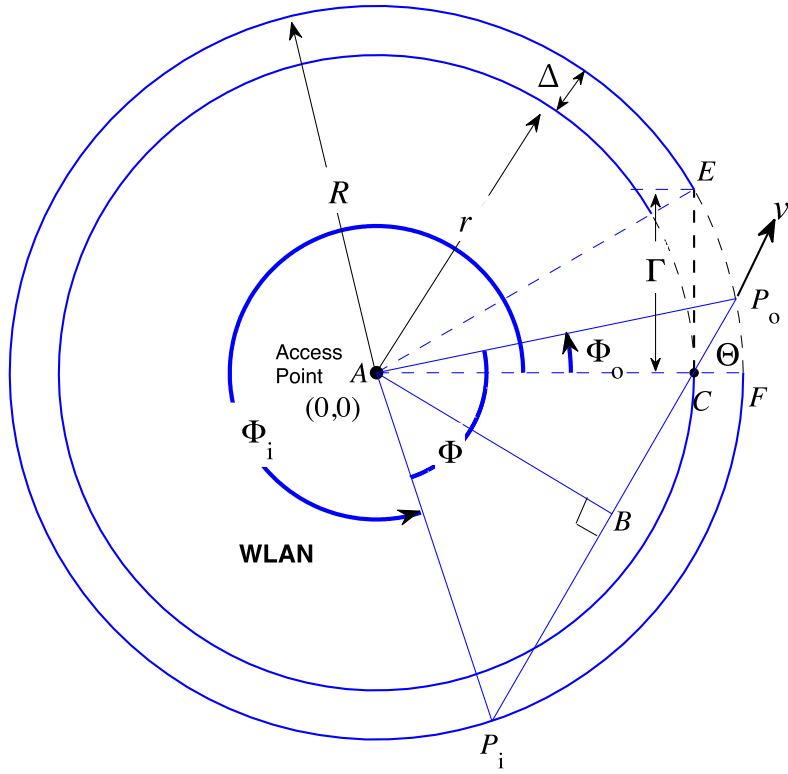

Fig. 1. Geometry and mobility parameters of the model.

direction $\Theta$ measured from $x$-axis. The distance between points $C$ and $P_{o}$ is the critical distance and is denoted as $D=\mathrm{m} \overline{C P_{o}}$. Depending on the value of $\Theta$, the smallest value of $D$ is equal to $\Delta$, in radial direction, when $\Theta=0$ and its largest value is equal to $\Gamma=\sqrt{R^{2}-r^{2}}$, in tangential direction, when $\Theta=\pi / 2$. In Fig. 1, we note that $\angle A C B$ and $\angle P_{o} C F$ are alternate angles; this gives $\mathrm{m} \overline{B C}=r \cos (\Theta)$ and $\mathrm{m} \overline{A B}=r \sin (\Theta)$. Applying Pythagoras theorem on $\triangle A B P_{o}$, we obtain a relationship between $D$ and $\Theta$

$$
D=\sqrt{R^{2}-r^{2} \sin ^{2}(\Theta)}-r \cos (\Theta)
$$

An expression, equivalent to (1), was obtained in [3] as follows:

$$
D=\sqrt{\frac{R^{2}\left(1+\Omega^{2}\right)+r^{2}\left(1-\Omega^{2}\right)-2 r \sqrt{R^{2}\left(1+\Omega^{2}\right)-r^{2} \Omega^{2}}}{1+\Omega^{2}}}
$$

where $\Omega=\tan (\Theta)$. The above expression, however, is complicated and does not help in analysis.

\subsection{Probability Density of Angle of Departure $(\Theta)$}

Owing to [4], we assume that angles of arrival $\left(\Phi_{i}\right)$ and departure $\left(\Phi_{o}\right)$ for an MT in a WLAN coverage area are uniformly distributed. We have $0 \leq$ $\Phi_{i}, \Phi_{o} \leq 2 \pi$. The interior angle, opposite to the traveling path $\overline{P_{i} P_{o}}$, is denoted as $\Phi$ and can be expressed as $\Phi=\min \{\tilde{\Phi}, 2 \pi-\tilde{\Phi}\}$, where $\tilde{\Phi}=$ $\left|\Phi_{o}-\Phi_{i}\right|$. For any given value of $\Phi_{i}$ and $\Phi_{o}$ in $[0,2 \pi]$, the random variable $\Phi$ can only have values in the range $[0, \pi]$ (this is also reported in $[5$, page 967]). The pdf of $\Phi$ is obtained as:

$$
f_{\Phi}(\phi)=\frac{2}{\pi}\left(1-\frac{\phi}{\pi}\right), \quad 0 \leq \phi \leq \pi
$$

The relation between the random variables $\Phi$ and $\Theta$ is

$$
\cos \left(\frac{\Phi}{2}\right)=\frac{r}{R} \sin (\Theta)
$$


In the upper semicircle, $\Theta$ lies in the range $[0, \pi / 2]$, it gives $\Phi \in\left[2 \cos ^{-1}(r / R)\right.$, $\pi]$. Denoting $\eta \equiv r / R$, the pdf of $\Phi$, under the condition $\Phi>2 \cos ^{-1}(r / R)$, is given as:

$$
\begin{array}{r}
f_{\Phi}\left(\phi \mid \phi>2 \cos ^{-1}(\eta)\right)=\frac{f_{\Phi}(\phi)}{1-F_{\Phi}\left(2 \cos ^{-1}(\eta)\right)} \\
\text { for } 2 \cos ^{-1}(\eta) \leq \phi \leq \pi
\end{array}
$$

We evaluate the cdf $F_{\Phi}\left(2 \cos ^{-1}(\eta)\right)$ as follows:

$$
\begin{aligned}
F_{\Phi}\left(2 \cos ^{-1}(\eta)\right) & =\operatorname{Prob}\left[\Phi \leq 2 \cos ^{-1}(\eta)\right] \\
& =\int_{0}^{2 \cos ^{-1}(\eta)} \frac{2}{\pi}\left(1-\frac{\phi}{\pi}\right) \mathrm{d} \phi \\
& =\frac{4}{\pi}\left(\cos ^{-1}(\eta)-\frac{\left(\cos ^{-1}(\eta)\right)^{2}}{\pi}\right)
\end{aligned}
$$

The pdf of $\Theta$ is given as:

$$
f_{\Theta}(\theta)=\sum_{k} \frac{f_{\Phi}\left(\phi_{k} \mid \phi_{k}>2 \cos ^{-1}(\eta)\right)}{\left|g^{\prime}\left(\phi_{k}\right)\right|}
$$

where $\Theta \equiv g(\Phi)=\sin ^{-1}\left(\frac{R}{r} \cos \left(\frac{\Phi}{2}\right)\right)$. Since $\Phi$ ranges from 0 to $\pi$, we have a single root, $\phi_{k}=2 \cos ^{-1}(r \sin (\theta) / R)$, in the semicircle for the relation (3). The derivative of $g(\phi)$ is

$$
\begin{aligned}
g^{\prime}\left(\phi_{k}\right) & =\left.\frac{\mathrm{d}}{\mathrm{d} \phi} \sin ^{-1}\left(\frac{R}{r} \cos \left(\frac{\phi}{2}\right)\right)\right|_{\phi=\phi_{k}} \\
& =\left.\frac{-R}{2 r} \frac{\sin \left(\frac{\phi_{k}}{2}\right)}{\sqrt{1-\frac{R^{2}}{r^{2}} \cos ^{2}\left(\frac{\phi_{k}}{2}\right)}}\right|_{\phi_{k}=2 \cos ^{-1}(r \sin (\theta) / R)} \\
& =-\frac{\sqrt{R^{2}-r^{2} \sin ^{2}(\theta)}}{2 r \cos (\theta)}
\end{aligned}
$$

Substituting values of $f_{\Phi}\left(\phi_{k} \mid \phi_{k}>2 \cos ^{-1}(\eta)\right)$ and $g^{\prime}\left(\phi_{k}\right)$, we obtain

$$
f_{\Theta}(\theta)=\frac{4 \eta \cos (\theta)\left(\pi-2 \cos ^{-1}(\eta \sin (\theta))\right)}{\left(\pi-2 \cos ^{-1}(\eta)\right)^{2} \sqrt{1-\eta^{2} \sin ^{2}(\theta)}}, \quad 0 \leq \theta \leq \frac{\pi}{2}
$$

\subsection{Probability Density of Critical Distance (D)}

The distance that an MT would traverse in the WLAN coverage area after the handover trigger is $D$ and it is a function of $\Theta$. From (1), we have $D=g(\Theta)$, which shows that $D$ increases monotonically with $\Theta$ and this yields a single root of function $g^{-1}(\Theta)$, viz.

$$
\Theta=g^{-1}(\Theta)=\cos ^{-1}\left(\frac{R^{2}-r^{2}-D^{2}}{2 r D}\right)
$$

After simple manipulations, the pdf of $D$ is obtained as:

$$
f_{D}(d)=\frac{4\left(\Gamma^{2}-d^{2}\right)\left(\pi-2 \cos ^{-1}\left(\eta \sqrt{1-\left(\frac{\Gamma^{2}-d^{2}}{2 r d}\right)^{2}}\right)\right)}{\left(\pi-2 \cos ^{-1}(\eta)\right)^{2} d \sqrt{4 r^{2} d^{2}-\left(\Gamma^{2}-d^{2}\right)^{2}}},
$$




\subsection{Probability of Handover Failure}

The probability of handover failure is given by

$$
P_{\mathrm{f}}=\left\{\begin{array}{lr}
1, & v \tau>\Gamma \\
\operatorname{Prob}[D<v \tau], & \Delta \leq v \tau \leq \Gamma \\
0, & v \tau<\Delta
\end{array}\right.
$$

where $\tau$ is the handover latency. We obtain for $\Delta \leq v \tau \leq \Gamma$

$$
\begin{aligned}
P_{\mathrm{f}} & =\int_{\Delta}^{v \tau} f_{D}(\lambda) \mathrm{d} \lambda \\
& =\frac{\pi^{2}-4\left(\pi-\cos ^{-1}\left(\frac{r}{R} \sqrt{1-\Lambda^{2}}\right)\right) \cos ^{-1}\left(\frac{r}{R} \sqrt{1-\Lambda^{2}}\right)}{\left(\pi-2 \cos ^{-1}\left(\frac{r}{R}\right)\right)^{2}}
\end{aligned}
$$

where $\Lambda=\left(\Gamma^{2}-v^{2} \tau^{2}\right) /(2 r v \tau)$. From (12), we can obtain the value of $r$ for a desired value of $P_{\mathrm{f}}$. Equation (12), however, is a nonlinear equation in $r$; a closed form solution may not be possible to acquire. Moreover, numerical methods can be used to calculate $r$. We use bisection numerical method to solve for $r$. Once $r$ is calculated, the corresponding value of $S_{\text {ho }}$ is calculated using the path loss model. Depicted in Fig. 2 is the plot of critical radius $r$ versus speed $v$ for $P_{\mathrm{f}}=0.02,0.05,0.1$ and 0.2 . This shows that the value of $r$ decreases as the $P_{\mathrm{f}}$ decreases for a particular value of $v$. This is because the handover must be started earlier to maintain desired $P_{\mathrm{f}}$.

The received signal strength $S_{l}$ (in $\mathrm{dBm}$ ) experienced by an MT at distance $l$ away from the AP (center) inside the WLAN coverage area is expressed using the log-distance path loss model [6, Chap. 2]: $S_{l}=S_{\text {ref }}+$ $10 \beta \log _{10}\left(R_{\text {ref }} / l\right)+X_{\sigma}$, where $l$ is the radial distance between the AP and MT, $R_{\text {ref }}$ is the distance between the AP and a reference point, $S_{\text {ref }}$ is the signal strength at the reference point in $\mathrm{dBm}, \beta$ is the path loss exponent ( $\beta=4$ for our simulation), and $X_{\sigma}$ is a Gaussian distributed random variable with zero mean and standard deviation $\sigma$ (typical value of $\sigma$ is 6 to $8 \mathrm{~dB}$ ). We can relate $S_{\text {ho }}$ and $S_{\mathrm{qt}}$ as follows:

$$
S_{\mathrm{ho}}=S_{\mathrm{qt}}+10 \beta \log _{10}\left(\frac{R}{r}\right)+X_{\sigma}
$$

where $r$ is the root of the Equation (12). Fig. 3 shows that the value of $S_{\text {ho }}$ increases as the speed increases for a particular value of $\tau$. This is because the handover must be started earlier for the fast moving users. Similarly, for a particular speed value, $S_{\text {ho }}$ increases as $\tau$ increases; this is to initiate an early handover for higher values of $\tau$. Here we also compare our results with those of [1] where $\Theta$ was assumed to be uniformly distributed. The similar performance of these two different methods reveals that how robust these algorithms are to different pdfs. This would be important in practice, as in the real world, the angle of departures are not going to be known.

Once $S_{\mathrm{ho}}$ is calculated, the handover trigger unit monitors the signal strength from the AP and sends a trigger to handover execution unit to start the registration procedures with target network when signal strength from the AP drops below $S_{\mathrm{ho}}$. 


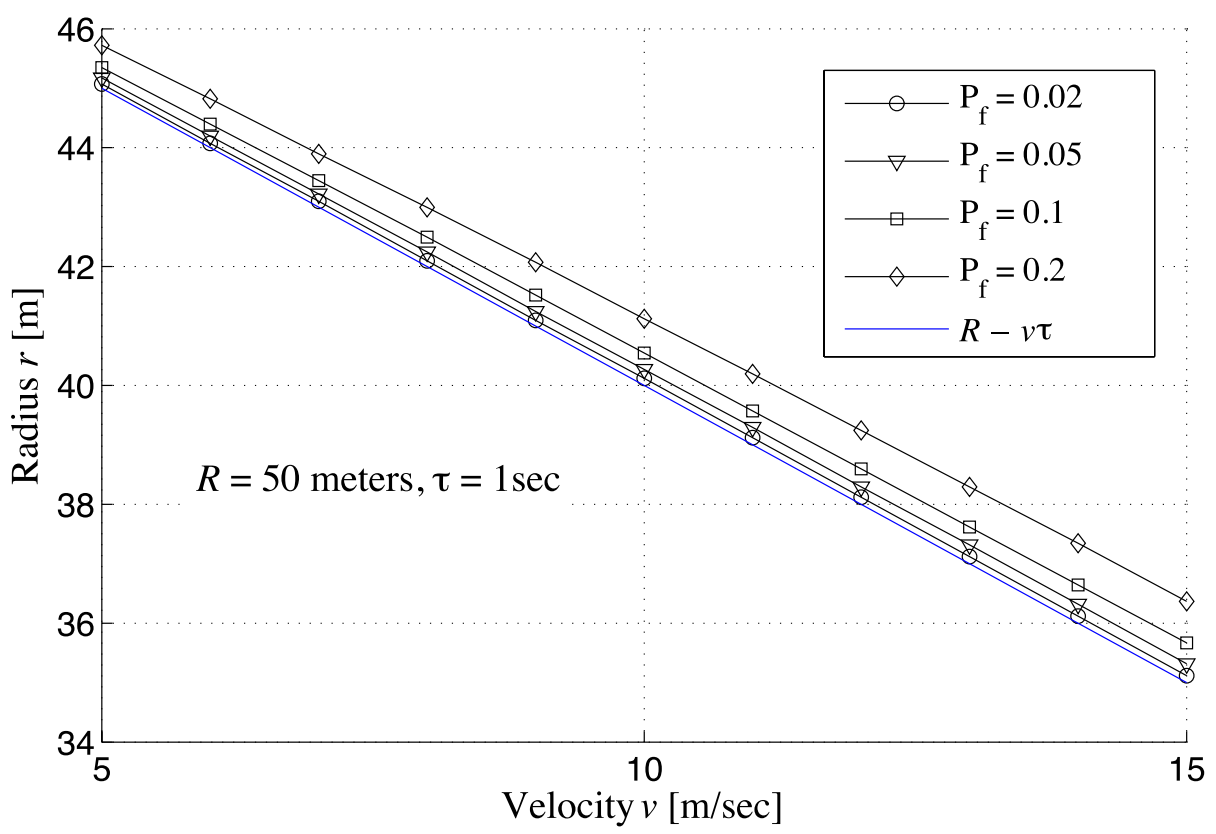

Fig. 2. The value of $r$ vs. speed for different values of $P_{\mathrm{f}}$.

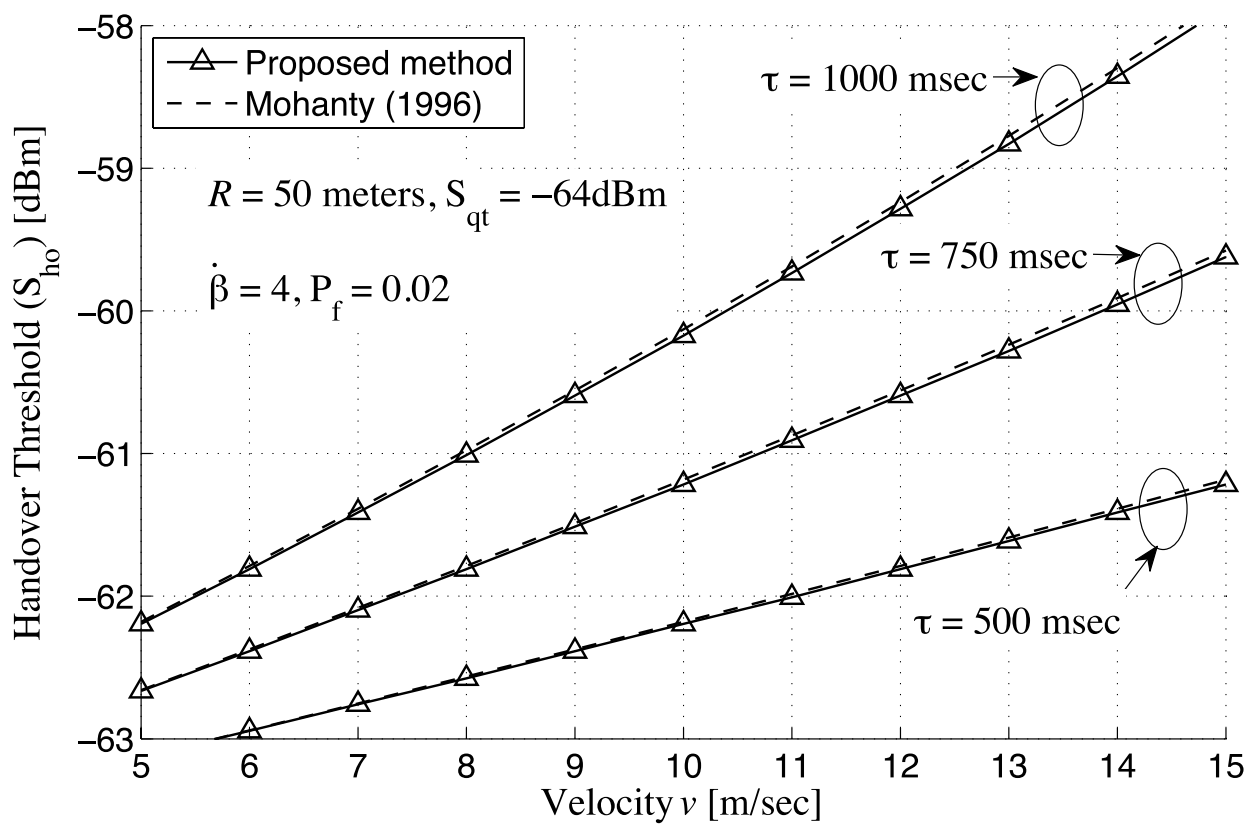

Fig. 3. The value of $S_{\text {ho }}$ vs. speed for different values of $\tau$.

\section{Simulation Results}

To analyze the probability of handover failures for the proposed and fixed received signal strength (FRSS) based handover triggering condition estimation methods, we assume that the coverage radius of the WLAN cell $(R)$ is $50 \mathrm{~m}$, the handover latency $(\tau)$ is $1 \mathrm{sec}$, and the value of $S_{\mathrm{qt}}$ is taken as $-64 \mathrm{dBm}[1]$. For each velocity value, every $P_{\mathrm{f}}$ trace is an ensemble average of 10,000 independent realizations of $\Theta$. In Fig. 4, we depict plots of $P_{\mathrm{f}}$ exhibited by FRSS for three different values of $S_{\text {ho }}$. Note that FRSS is able to maintain $P_{\mathrm{f}} \approx 0$ up to certain values of velocity; after that, it rises rapidly. 


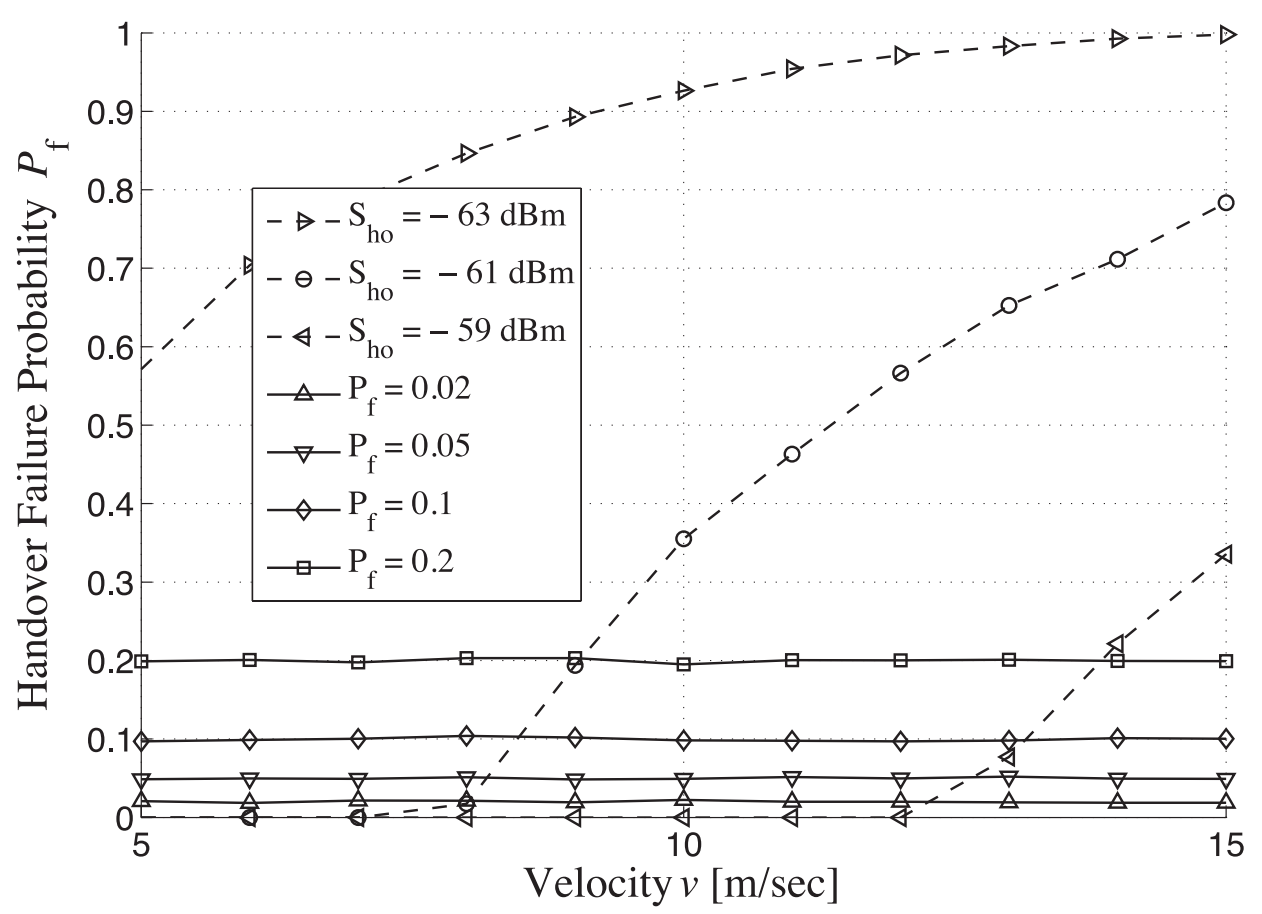

Fig. 4. Performance of proposed and FRSS methods.

We also depict plots of $P_{\mathrm{f}}$ exhibited by the proposed method versus velocity for four different target values of $P_{\mathrm{f}}$. It can be observed that, regardless the velocity of MT, the proposed method is capable of maintaining the target $P_{\mathrm{f}}$ at its desired value. In practical sense, the use of proposed method would result in maximized WLAN usage for a given probability of failure. 